ARTÍCULOS ORIGINALES

Rev Chil Salud Pública 2014; Vol 18 (3): 249-256
Guadalupe Guerrero

JosÉ LÓPEZ

Nuri VilLAseñor

César Gutiérrez

Docente investigador Facultad de Medicina Universidad Autónoma de Querétaro lupitag|@uaq.mx

Yazmín Sánchez

Luis Santiago

Omar Martínez

Estudiante Maestría en Investigación Médica. Facultad de Medicina Universidad Autónoma de Querétaro

Nayely Maya Lozano

Pasante de Licenciatura Nutrición Universidad Autónoma de Querétaro

\section{DISEÑO Y VALIDACIÓN DE UN CUESTIONARIO DE HÁBITOS DE VIDA DE ALIMENTACIÓN Y ACTIVIDAD FÍSICA PARA ESCOLARES DE 8-12 AÑOS*}

\author{
Design AND VALIDATION OF A QUESTIONNAIRE TO ASSESS HEALTH \\ HABITS OF SCHOOL CHILDREN AGED 8-12
}

\section{RESUMEN}

Introducción: El sobrepeso y la obesidad son actualmente un serio problema de salud pública en México, principalmente en la etapa infantil, según la Encuesta Nacional de Salud y Nutrición (ENSANUT, 2012). La obesidad es una enfermedad multifactorial, en la cual se involucran aspectos genéticos, ambientales $y$ de estilo de vida. Para prevenir y tratarla deben modificarse los hábitos de vida, mediante promoción y educación para la salud. Para cambiar los hábitos de la población primero se debe conocer cuáles son, posteriormente crear estrategias para modificarlos. No existen cuestionarios para identificar los hábitos de vida saludables en niños escolares en nuestro medio.

Objetivo: Diseñar y evaluar la fiabilidad y validez del cuestionario.

Métodos: Se aplicó en 417 escolares. Se usaron medidas de tendencia central y dispersión para los dominios y grupos; consistencia interna mediante alpha de Cronbach y correlación interescalar.

Resultados: Se encontró que los 27 ítems en los dos dominios superaron el estándar propuesto de fiabilidad ( $\alpha$ de Cronbach) mayor a 0.50. Se encontró correlación aceptable, en las diferentes dimensiones del cuestionario (total vs. dominios). La estabilidad test-retest es superior a 0.75 en las dos dimensiones; en los análisis de validez efectuados (de contenido y constructo) los resultados son satisfactorios.

Conclusiones: La versión del cuestionario reúne los criterios de consistencia interna y validez de contenido y constructo. Dichos resultados ponen de manifiesto que el cuestionario propuesto pueda ser utilizado como una herramienta para valorar los hábitos de vida en escolares.

Palabras Clave: hábitos de vida, validación de cuestionarios, hábitos alimentarios, hábitos de actividad física, encuestas nutricionales, atención primaria de salud.

\footnotetext{
* Proyecto financiado por Fondo Mixto CONACYT - Gobierno del Estado de Querétaro en la convocatoria 2011-02 "Diseño y evaluación de un programa educativo para fomentar hábitos de vida saludables en la prevención de la obesidad infantil”.
} 


\section{ABSTRACT}

Introduction: Being over weight and obesity are currently serious public bealth problems in Mexico, particularly in childhood. According to the National Health and Nutrition Examination Survey (ENSANUT, 2012), obesity is a multifactorial disease in which genetic, environmental and lifestyle issues are involved. To prevent and treat obesity lifestyle should be altered through bealth promotion and education. To change the habits of the population, first they need to be recognised, then strategies to modify them developed. Presently, there are no questionnaires to identify healthy habits in school children in our area.

Objective: To design and evaluate the reliability and validity of a questionnaire that assesses bealth habits in children.

Methods: 417 schoolchildren undertook the questionnaire. Measures of central tendency and dispersion for domains and groups were used; internal consistency using Cronbach's alpha and interscalar correlation.

Results: It was found that the 27 items in the 2 domains exceeded the suggested standard of reliability (Cronbach $\alpha$ ) greater than 0.50 . Acceptable correlation was found in the different dimensions of the questionnaire (total vs. domains). The test-retest stability was higher than 0.75 in both dimensions; in analyses performed on validity (content and construct) the results were satisfactory.

Conclusions: The version of the questionnaire met the criteria of internal consistency and content validity and construct. These results show that the proposed questionnaire can be used as a tool to assess lifestyle in school children.

Keywords: Lifestyle habits, validating questionnaires, dietary habits, physical activity habits, nutritional surveys, primary bealth care.

\section{INTRODUCCIÓN}

El sobrepeso y la obesidad son actualmente un serio problema de salud pública en México y muchos otros países del mundo, principalmente en la etapa infantil, constituyéndose como una epidemia silenciosa. La obesidad y el sobrepeso se definen como una acumulación anormal o excesiva de grasa. ${ }^{1}$ Según los resultados de la Encuesta Nacional de Salud y Nutrición (ENSANUT) realizada en 2012, y su comparación con lo obtenido en la Encuesta Nacional de Nutrición (ENN) en 1999, las cifras de sobrepeso y obesidad en niños de 5 a 11 años subió de $22,8 \%$ en 1999 a 36,9\%, y de $25,5 \%$ a $32 \%$ en niñas. Los niños en edad escolar (ambos sexos), de 5 a 11 años, presentaron una prevalencia nacional combinada de sobrepeso y obesidad en 2012 de 34,4\%, $19,8 \%$ para sobrepeso y $14,6 \%$ para obesidad. ${ }^{2}$

La obesidad es una enfermedad multifactorial, crónica, en la cual se involucran aspec- tos genéticos, ambientales y de estilo de vida. Se caracteriza por un balance positivo de energía, que ocurre cuando la ingestión de calorías excede al gasto energético, ocasionando un aumento en los depósitos de grasa corporal y, por ende, ganancia de peso..$^{3-6}$

La obesidad es el principal factor de riesgo para el desarrollo de diabetes tipo 2, enfermedades cardiovasculares (especialmente las cardiopatías y los accidentes vasculares cerebrales), hipertensión arterial, dislipidemias, enfermedades osteoarticulares y algunos tipos de cáncer, entre ellos el de mama y próstata. En los niños, la obesidad infantil se asocia a una mayor probabilidad de muerte prematura, así como de obesidad y discapacidad en la edad adulta. ${ }^{7-9}$

Se ha sugerido mayor susceptibilidad genética a la obesidad en población mexicana comparada con otras. Sin embargo, los factores genéticos no explican los aumentos inusitados de la prevalencia de obesidad en 
la población mexicana en un periodo tan corto. ${ }^{6,10}$ Este gran aumento en la prevalencia de obesidad parece obedecer a cambios en los factores ambientales, debidos principalmente a la transición epidemiológica en la que México se encuentra actualmente. ${ }^{11-13} \mathrm{La}$ escasa actividad física de las personas, es la consecuencia del cambio de patrones de conducta que derivan hacia modos de vida más sedentarios, tanto en el trabajo como en el tiempo de ocio. En la población infantil estos fenómenos se agudizan, por el incremento de horas que dedican a ver la televisión y al tiempo destinado a la computadora y/o videojuegos. La vida en las grandes ciudades se ha convertido en obstáculo para que los niños y niñas jueguen en las calles y jardines, puedan desplazarse a pie o ir en bicicleta. ${ }^{14,15}$

Ante esto, se ha señalado la necesidad de educar en alimentación saludable, considerando estrategias innovadoras y pertinentes de intervenciones educativas que involucren a los niños y su entorno. Para ello es fundamental identificar los conocimientos y hábitos alimentarios en escolares, con el objetivo de elaborar una estrategia con materiales educativos que permitan desarrollar hábitos saludables. ${ }^{16,17}$

Actualmente en México no existe un instrumento para identificar los hábitos de vida en niños escolares, por lo tanto es necesario contar con este tipo de instrumentos, que puedan utilizarse para medir el impacto de estrategias educativas, con el objetivo de prevenir el sobrepeso y la obesidad. En ese contexto se presenta el siguiente estudio, que pretende elaborar un instrumento que permita medir los hábitos de vida de alimentación y actividad física en escolares de 8-12 años de edad, de escuelas públicas de la ciudad de Querétaro.

\section{MATERIAL Y MÉTODOS}

El presente estudio es un diseño no experimental de tipo transversal, para validación de cuestionarios. Después de revisar la literatura sobre la metodología para la construcción y validación de cuestionarios, conceptos y de- finición de hábitos de vida, dominios que lo comprenden, los investigadores del proyecto elaboraron una primera versión del cuestionario autoadministrado con 35 ítems y siete opciones de respuesta, para identificar los hábitos de vida, en base a una amplia revisión bibliográfica. La validez de contenido se evaluó mediante consenso de expertos, un panel multidisciplinario de ocho profesionales de la salud conformado por médicos pediatras (2), nutriólogos (2), psicólogo infantil, médico familiar, experto en diseño y validación de cuestionarios y un redactor de estilo, todos ellos con más de cinco años de experiencia en su ramo, quienes evaluaron el instrumento de manera independiente y ciega.

De esta primera versión se eliminaron 10 ítems, finalmente se agregaron dos ítems más de acuerdo a propuestas de los expertos, hasta unificar criterios. Se creó entonces la tercera versión del instrumento que consta de 27 ítems, cada uno de ellos, por sugerencia de los expertos con cinco opciones de respuestas, que se traducen a números, del 1 al 5 . Si la opinión es favorable a la actitud que deseamos medir, el diariamente se codificará con un 5 y el nunca o menos de una vez por mes con un $1, \mathrm{y}$ si es desfavorable el diariamente se codificará con un 1 y el nunca o menos de una vez por mes con un 5 , y luego sumaremos todas las respuestas, de modo que la puntuación más alta indique siempre la conducta más saludable. Por lo tanto la puntuación total mínima serán 27 puntos y la máxima 135. Cada uno de los ítems tiene el mismo valor, no hay ítems ponderados.

El tamaño de la muestra fue de 405 y se calculó considerando la inclusión de 15 sujetos por cada ítem del instrumento, número necesario para efectuar análisis factorial. ${ }^{18,19}$ Se aumentó un 5\% más el tamaño de la muestra, para evitar pérdidas (20 niños), ocho de ellos tenían cuestionarios incompletos. Finalmente se estudió una muestra no probabilística de 417 escolares de 8 a 12 años de edad, de tercero a sexto de primaria, de ambos géneros, que supieran leer y escribir, de una escuela de la ciudad de Querétaro, asignada por la USEBEQ (Unidad de Servicios para la Educación Básica en el Estado de Querétaro). Todos los 
alumnos fueron informados sobre la finalidad del estudio y se describió y explicó sobre el procedimiento de llenado del instrumento. El estudio contó con la aprobación del comité de bioética. Los padres y tutores de los niños llenaron y firmaron la carta de consentimiento informado para autorizar la aplicación del instrumento.

Los participantes contestaron el cuestionario de manera autoadministrada en sus aulas de clase y regresamos tres semanas después para contestar el instrumento por segunda vez, considerando este lapso lo suficientemente prolongado como para que no recordaran las respuestas dadas en la primera administración y lo suficientemente corto como para no presentar cambios importantes en el estilo de vida. Lapsos similares se han utilizado para evaluar la consistencia test-retest de diferentes instrumentos; para medir la estabilidad se usó el coeficiente de correlación de Pearson, entre los primera y la segunda aplicación. ${ }^{20} \mathrm{El}$ análisis de consistencia interna total y por dimensión se realizó mediante la aplicación del coeficiente alpha de Cronbach, basado en la varianza de los ítems del instrumento. Para estudiar la interrelación de las preguntas que conforman las dimensiones en relación al puntaje de la dimensión y del total del puntaje, se utilizaron coeficientes de correlación de Pearson, la estructura matricial se construyó a través de un análisis factorial usando como método de extracción al análisis de Componentes Principales, aplicando la metodología Varimax.

Para determinar los puntos de corte del puntaje resultante del instrumento, se utilizaron los valores de percentil 25, entre percentil 25 y percentil 75 y mayor a percentil 75 . Para comparar los puntajes respecto a género se aplicó la prueba de $t$ de Student. Se usó el programa estadístico SPSS 17.0.

\section{RESULTADOS}

El cuestionario se aplicó a 417 estudiantes de primaria de tercero a sexto grado. La muestra estudiada tenía una media de edad de 10.0 años. El 51,1\% (213) eran de sexo femenino y $48,9 \%$ (204) de sexo masculino.
La tarea de los miembros del panel de expertos fue examinar cada uno de los enunciados del cuestionario y determinar cuáles debían permanecer por ser relevantes para nuestro estudio y cuáles debían ser eliminados por la razón opuesta. Al final del proceso permanecieron 25 de las preguntas originales y fueron eliminadas 10 . De las 25 que se mantuvieron, se sugirió realizar algunas adecuaciones a cinco de ellas. De la misma forma, se sugirió agregar dos nuevas preguntas. Por sugerencia de los expertos se redujeron a cinco las opciones de respuesta. Al evaluar la fiabilidad de las preguntas que formaron parte del instrumento, se encontró un alpha de Cronbach de 0.81. La dimensión alimentación y nutrición, que constó de 18 ítems, tenía una confiabilidad ligeramente menor, con valores de alpha de Cronbach de 0.79 y la dimensión actividad e inactividad física, que constaba de nueve ítems (alpha de Cronbach de 0.76). Lo anterior implica mencionar que al utilizar las dimensiones por separado disminuye la confiabilidad, por lo que se sugiere la aplicación total del instrumento. La estabilidad o consistencia temporal de las dos dimensiones se valoró mediante la readministración del cuestionario a 105 de los 417 escolares iniciales, debido a que muchos padres de familia no aceptaron una segunda aplicación del cuestionario. Los coeficientes de correlación de cada una de las dos dimensiones fueron superiores a 0.75 , y en la correlación test-retest del total del instrumento se encontró una $\mathrm{r}$ de 0.82 .

Se recomienda un análisis factorial cuando el estadístico Kaiser-Meyer-Olkin (KMO) tiene un valor $>0.5$, aunque es óptimo si el $\mathrm{KMO}$ es mayor a 0.7 , agregando la prueba de esfericidad de Bartlett significativo, lo que demuestra correlación entre los datos. En este caso el valor de KMO fue de 0.768 y la prueba de esfericidad de Barlett tuvo un valor significativo $(\mathrm{p}=0.000)$. Dichos resultados nos permitieron realizar un análisis factorial, que nos admitió agrupar en las dos dimensiones, que se conformaron mediante el método varimax, Estas dos dimensiones pudieron explicar el $63,2 \%$ de la varianza.

Al estudiar las correlaciones de cada una de las preguntas respecto a su dimensión y al 
Tabla 1. Correlación de Pearson Ítem Dimensión. Ítem Total de la estructura factorial Dimensión actividad e inactividad física * $\mathrm{p}<0.05$

\begin{tabular}{ccc}
\hline Preguntas & Ítem dimensión & Ítem total \\
\hline 1 & $0.692 *$ & $0.356^{*}$ \\
4 & $0.741 *$ & $0.339 *$ \\
7 & $0.767 *$ & $0.626^{*}$ \\
10 & $0.522^{*}$ & $0.426^{*}$ \\
13 & $0.672 *$ & $0.356^{*}$ \\
16 & $0.726 *$ & $0.474 \%$ \\
19 & $0.661 \%$ & $0.376 *$ \\
22 & $0.639 *$ & $0.505^{*}$ \\
25 & $0.728 *$ & $0.585^{*}$ \\
\hline
\end{tabular}

Tabla 2. Correlación de Pearson Ítem Dimensión. Ítem Total de la estructura factorial. Dimensión alimentación y nutrición * p $<0.05$

\begin{tabular}{ccc}
\hline Preguntas & Ítem dimensión & Ítem total \\
\hline 2 & $0.766^{*}$ & $0.409^{*}$ \\
3 & $0.639^{*}$ & $0.505^{*}$ \\
5 & $0.733^{*}$ & $0.326^{*}$ \\
6 & $0.701^{*}$ & $0.359^{*}$ \\
8 & $0.642^{*}$ & $0.365^{*}$ \\
9 & $0.669^{*}$ & $0.376^{*}$ \\
11 & $0.797^{*}$ & $0.637^{*}$ \\
12 & $0.630^{*}$ & $0.307^{*}$ \\
14 & $0.827^{*}$ & $0.318^{*}$ \\
15 & $0.713^{*}$ & $0.461^{*}$ \\
17 & $0.694^{*}$ & $0.449^{*}$ \\
18 & $0.572^{*}$ & $0.306^{*}$ \\
20 & $0.733^{*}$ & $0.326^{*}$ \\
21 & $0.661^{*}$ & $0.376^{*}$ \\
23 & $0.585^{*}$ & $0.515^{*}$ \\
24 & $0.627^{*}$ & $0.318^{*}$ \\
26 & $0.726^{*}$ & $0.464^{*}$ \\
27 & $0.720^{*}$ & $0.322^{*}$ \\
\hline
\end{tabular}

total del instrumento, se observa que están correlacionadas significativamente. No se encontraron correlaciones menores a $0.300(\mathrm{Ta}-$ bla 1 y 2 ).

El puntaje total de las dos dimensiones del instrumento, permite clasificar de acuerdo a tres puntos de corte. Es así como a aquellos que tengan menos de 95 puntos, se les clasificará con hábitos alimentarios deficientes, ya que el estilo de vida del niño plantea muchos factores de riesgo. A quienes presenten un puntaje dentro del rango entre los 95 a 109 puntos, se les considerará con hábitos alimentarios suficientes, que nos indica que el estilo de vida tiene beneficios para la salud, aunque también presenta riesgos. Mientras quienes presenten un puntaje superior a 109 puntos, se les catalogará como personas con hábitos alimentarios saludables, ya que el estilo de vida representa una influencia adecuada para la salud. Se proponen las preguntas del instrumento final; las preguntas $1,4,7,10,13$, 16, 19, 22 y 25 miden la dimensión actividad física, y las preguntas 2, 3, 5, 6, 8, 9, 11, 12, 14, $15,17,18,20,21,23,24,26$ y 27 , cada una con cinco opciones de respuesta: Nunca o menos de 1 vez por mes, 1 a 3 veces por mes, 1 o 2 veces por semana, 3 a 6 veces por semana $\mathrm{y}$ diariamente (Anexo 1).

\section{DISCUSIÓN}

La medición del constructo "hábitos de vida" es una tarea difícil debido a las dificultades que implica su medición directa de manera objetiva. El presente cuestionario fue diseñado con el objetivo de identificar los hábitos de vida saludables, de alimentación y actividad física en escolares de 8 a 12 años de edad. La consistencia interna de los ítems y de los dominios medida por el alfa de Cronbach fue mayor a 0.50. De acuerdo con Hill y Hill, ${ }^{21}$ "un $\alpha$ de Cronbach entre 0,7 y 0,8 se puede considerar aceptable. De esta manera los coeficientes de fiabilidad, como en la mayoría de las estadísticas descriptivas, varían de una muestra a otra y tienen que ser considerados como estimaciones. Otros autores afirman que un $\alpha$ de Cronbach superior a 0,5, en estudios de validación de cuestionarios puede ser considerado como aceptable..$^{21,22}$

En este caso todos los ítems del cuestionario han presentado aceptable y buena consistencia interna por el $\alpha$ de Cronbach, superior a 0,76 ítem total. En este caso se obtiene una elevada estabilidad de las puntuaciones, de acuerdo a la correlación de Pearson en el testretest. Si los niveles de hábitos de vida de los niños no han variado a lo largo de las tres semanas transcurridas entre las dos aplicaciones, podemos decir que el test proporciona 
bastantes garantías respecto a la precisión con la que mide. El análisis de los ítems, agrupados en los dominios, permite observar que todos ellos están contribuyendo a la estabilidad del instrumento. La correlación de Pearson ítem-dimensión fue $>0.522$; la correlación ítem-total de ítems fue $>0.306$.

Los resultados de este estudio sugieren que el instrumento posee una buena confiabilidad y estabilidad. Si hay cambios en las variables que determinan los hábitos de vida, los escolares podrían ser debidamente reclasificados. Esto es de suma importancia para los programas de intervención, cuyo propósito es promover el estilo de vida como una forma de mejorar la salud y la calidad de vida. Cambios positivos en la clasificación según el cuestionario indicarán que el programa de intervención está alcanzando los resultados esperados. ${ }^{23}$ Como se planteaba al comienzo del trabajo, el diseño de un instrumento como un cuestionario no es fácil y todos los intentos de mejorar la validez y fiabilidad son pocos, $y$ eso nos permite señalar algunas limitaciones del estudio; entre ellas está principalmente la selección no probabilística de la muestra, que nos sugiere su falta de representatividad, además de pertenecer a un solo centro escolar; lo cual nos obliga a realizar más estudios que nos permitan el uso definitivo del instrumento, estudios que incluyan la validez de criterio, ya que cuando se diseña un nuevo instrumento de medición se dispone de algún método alternativo de medición del fenómeno estudiado con validez demostrada, que se toma como referencia para determinar la validez del nuevo instrumento, y que en el presente estudio no se pudo realizar.

\section{REFERENCIAS}

1. Aguilar H, Pérez P, Díaz de León E, CobosAguilar H. Factores Psicosociales asociados a sobrepeso y obesidad en niños de 8-15 años. Rev. Pediatría de México. 2011; 13(1): 17-23.

2. Encuesta Nacional de Salud y Nutrición. 2012. Estado de nutrición, anemia, seguridad alimentaria en la población mexicana [en línea] México: INSP; 2012 [consultado
23.12.2012]. Disponible en: http://ensanut. insp.mx/doctos/ENSANUT2012_Nutricion.pdf

3. Fernández S, Montoya Y, Viguri R. Sobrepeso y obesidad en menores de 20 años de edad en México. Bol Med Hosp Infant Mex. 2011; 68(1): 71-73.

4. Bouchard C. Gene-environment interactions in the etiology of obesity: defining the fundamentals. Obesity (Silver Spring). 2008; 16 Suppl 3: S5-10.

5. Gardner D. The etiology of obesity. Mo Med. 2003; 100 (3): 242-7.

6. Weinsier RL, Hunter GR, Heini AF, Goran MI, Sell SM. The etiology of obesity: relative contribution of metabolic factors, diet, and physical activity. Am J Med. 1998; 105 (2): $145-50$.

7. Astrup A, Dyerberg J, Selleck M, Stender S. Nutrition transition and its relationship to the development of obesity and related chronic diseases. Obes Rev. 2008; 9 Suppl 1: 48-52.

8. Freeman-Fobbs P. Feeding our children to death: the tragedy of childhood obesity in America. J Natl Med Assoc. 2003; 95(2): 119.

9. Villanueva M, Hernández AM, Quiros A, Sánchez M. Prevalencia de obesidad infantil en niños entre 6 y 14 años de edad en una Unidad de Medicina Familiar del IMSS. Rev. Pediatría de México. 2011; 13(4): 151-154.

10. Barquera S, Campos-Nonato I, Rojas R, Rivera J. Obesidad en México: epidemiología y políticas de salud para su control y prevención. Gac Méd Méx. 2010; 146: 397-407.

11. López AMG, Rodríguez CM. Epidemiología y genética del sobrepeso y la obesidad. Perspectiva de México en el contexto mundial. Bol Med Hosp Infant Mex. 2008; 65: 421-429.

12. World Health Organization. Child growth standards: the WHO Child Growth Standards. The Who anthro study 2006 [en línea] WHO; 2014 [consultado 23.12.2012]. Disponible en: http://www.who.int/childgrowth/en/

13. Bacardí-Gascón M, Jiménez-Cruz A, Jones E, Guzmán-González V. Alta prevalencia de obesidad y obesidad abdominal en niños es- 
colares entre 6 y 12 años de edad. Bol Med Hosp Infant Mex. 2007; 64: 362-369.

14. Hernández-Herrera RJ, Salas-Rubio G, Castillo-Abdo JM, Saaib-Castillo JS, Tamez-Rodríguez VA, Dávila-Canales $\mathrm{R}$ et al. Prevalencia de sobrepeso y obesidad en 407 preescolares y escolares en el noreste mexicano. Med Univ. 2011; (51): 79-83.

15. Castañeda-Castaneira E, Molina-Frechero N, Ortiz-Pérez H. Sobrepeso-obesidad en escolares en un área marginada de la ciudad de México. Rev Mex Ped. 2010; 77: 241-247.

16. López E, MA. Etapas del cambio conductual ante la ingesta de frutas y verduras, control de peso y ejercicio físico de estudiantes de la Universidad del Desarrollo, sede Concepción, Chile. Rev Chil Nutr. 2008; 35(3): 215-24.

17. Lera L, Salinas J, Fretes G, Vio F. Validación de un instrumento para evaluar prácticas alimentarias en familias chilenas de escolares de 4 a 7 años. Nutr Hosp. 2013; 28(6): 1961-1970.

18. Morales P. El análisis factorial en la construcción e interpretación de tests, escalas y cuestionarios [en línea] Madrid: Universidad Pontificia Comillas; 2012 [consultado enero de 2013]. Disponible en: http://web. upcomillas.es/personal/peter/investigacion/ AnalisisFactorial.pdf
19. Morales P. Tamaño necesario de la muestra: ¿Cuántos sujetos necesitamos? Estadística aplicada a las Ciencias Sociales [en línea] Madrid: Universidad Pontificia Comillas, Madrid. 2012 [consultado enero de 2013]. Disponible en: http://www.upcomillas.es/personal/peter/investigacion/ Tama\%F1oMuestra.pdf

20. Pardo A, Ruiz M, Jódar E, Garrido J, De Rosendo JM, Usán LA. Desarrollo de un cuestionario para la valoración y cuantificación de los hábitos de vida relacionados con el sobrepeso y la obesidad. Nutr Hosp. 2004;19 (2): 99-109.

21. Hill MM, Hill A. Investigação por questionário. Lisboa: Silabo; 2000.

22. Hulme PA, Walker SN, Effle KJ, Jorgensen L, McGowan MG, Nelson JD, Pratt EN. Health promoting lifestyle behaviors of Spanish-speaking Hispanic adults. J Transcult Nurs. 2003; 14: 244-54.

23. Triviño-Quintero LP, Dosman-González V, Uribe-Vélez YL, Agredo-Zúñiga RA, JerezValderrama AM, Ramírez-Vélez R. Estudio del estilo de vida y su relación con factores de riesgo cardiovascular en adultos de mediana edad. Acta Med Colomb. 2009; 34: 158-163. 


\section{ANEXOS}

Ítems del cuestionario de hábitos de vida saludables de alimentación y actividad física (CHVSAAF) para escolares de 8-12 años

\begin{tabular}{|c|c|c|c|}
\hline NO. DE ÍTEM & DIMENSIÓN DE ALIMENTACIÓN Y NUTRICIÓN & PROMEDIO & $\begin{array}{c}\mathrm{DE} \\
\text { Desviación estándar }\end{array}$ \\
\hline 2. & Tomo refrescos o jugos embotellados & 3.23 & 1.18 \\
\hline 3. & Tomo leches saborizadas: chocolate, fresa, etc. & 3.23 & 1.49 \\
\hline 5. & Como frutas y verduras & 4.41 & 0.89 \\
\hline 6. & Desayuno antes de salir de mi casa & 4.67 & 0.98 \\
\hline 8. & Como pizzas o hamburguesas & 4.03 & 0.81 \\
\hline 9. & Como panes o pastelitos de paquete & 3.43 & 1.14 \\
\hline 11. & Si estoy aburrido o triste me da por comer & 3.65 & 1.42 \\
\hline 12. & $\begin{array}{l}\text { Hago } 5 \text { comidas al día (desayuno, lunch, comida, colación de } \\
\text { media tarde, cena) }\end{array}$ & 3.76 & 1.02 \\
\hline 14. & $\begin{array}{l}\text { Como algunas golosinas o frituras como: chicles, caramelos, } \\
\text { papas de bolsa, etc. }\end{array}$ & 3.32 & 1.06 \\
\hline 15. & Cuando tengo sed tomo agua sola & 4.1 & 1.23 \\
\hline 17. & $\begin{array}{l}\text { Sigo comiendo aunque ya no tenga hambre, solo por acabarme } \\
\text { lo que tengo en el plato. }\end{array}$ & 4.25 & 1.09 \\
\hline 18. & Como al menos 2 frutas al día & 4.07 & 1.23 \\
\hline 20. & Cuando veo la televisión como golosinas o frituras & 3.76 & 1.18 \\
\hline 21. & $\begin{array}{l}\text { El lunch que como todos los días lo compro en la escuela o } \\
\text { en la calle }\end{array}$ & 4.13 & 1.03 \\
\hline 23. & Si tengo hambre entre comidas, como una fruta & 3.26 & 1.52 \\
\hline 24. & A la hora de comer veo la televisión & 3.42 & 1.57 \\
\hline 26. & Como al menos 2 verduras al día & 3.99 & 1.29 \\
\hline \multirow[t]{2}{*}{27.} & $\begin{array}{l}\text { Consumo helados, malteadas, café o chocolate, preparados con } \\
\text { crema batida, cajeta [manjar] o chispas de chocolate }\end{array}$ & 3.68 & 1.11 \\
\hline & DIMENSIÓN ACTIVIDAD E INACTIVIDAD FÍSICA & & \\
\hline 1. & Hago actividades físicas y/o deportivas con mi familia & 3.18 & 1.18 \\
\hline 4. & $\begin{array}{l}\text { Estoy más tiempo en la computadora o videojuegos que jugan- } \\
\text { do fútbol u otro deporte o actividad física }\end{array}$ & 3.37 & 1.26 \\
\hline 7 & Me paso 2 horas o más viendo programas de televisión & 3.11 & 1.29 \\
\hline 10. & Juego en el parque, jardín o patio con otros niños & 3.41 & 1.35 \\
\hline 13. & A la hora de recreo hago algún deporte o actividad física & 3.29 & 1.33 \\
\hline 16. & $\begin{array}{l}\text { Practico algún tipo de actividad física o deportiva además de la } \\
\text { que realizo en la escuela los días de Educación Física }\end{array}$ & 3.88 & 1.16 \\
\hline 19. & Camino por lo menos 15 minutos por día & 3.94 & 1.27 \\
\hline 22. & Me paso una buena parte de la mañana o tarde acostado & 3.78 & 1.24 \\
\hline 25. & $\begin{array}{l}\text { Me quedo en casa sentado o acostado, en vez de jugar o hacer } \\
\text { deporte o actividad física }\end{array}$ & 3.68 & 1.11 \\
\hline
\end{tabular}

